\title{
PERCHÉ È COSİ DIFFICILE PER LA SCIENZA AFFRONTARE IL TEMA DELLA COSCIENZA E DEI SUOI RAPPORTI COL CERVELLO?
}

\author{
MAURO CERONI (*)
}

SuNTO. - È evidente a tutti, anche ai non addetti ai lavori, che la coscienza umana solleva un grande problema alla Scienza e quindi alle Neuroscienze. Tant'è vero che si cerca oggi in tutti i modi di eliminare la coscienza come puro epifenomeno o di ridurla completamente al cervello. L'articolo precisa innanzitutto il metodo scientifico e discute i possibili limiti di applicabilità al campo della coscienza. Cerca poi di mostrare la natura sfuggente ogni definizione della coscienza umana. Ricorda infine con una citazione dalla monumentale opera del Prof. Savoldi su "La Coscienza" un passaggio fondamentale che chiarisce al di là di ogni ambiguità, con l'aiuto di Pascal, l'unica modalità conoscitiva che permette di avvicinarsi alla coscienza e che non può essere il metodo sperimentale.

$* * *$

ABSTRACT. - It is evident to everyone, even to non-professionals, that human consciousness raises a major problem in Science and therefore in Neuroscience. So much so that today we try in every way to eliminate consciousness as pure epiphenomenon or to completely reduce it to the brain. The article specifies first the scientific method and discusses the possible limits of applicability to the field of consciousness. Then tries to show the elusive nature every definition of human consciousness has. Finally, it recalls a quote from the monumental work of Prof. Savoldi on "La Coscienza", a fundamental passage that clarifies beyond ambiguity, with the help of Pascal, the only cognitive modality (comprehension) that allows one to approach the conscience and which can not be the experimental method.

La scienza nasce nel momento in cui Galileo si accorge che per conoscere veramente, adeguatamente le cose, la realtà occorre stare davanti ad esse lealmente, fino in fondo, occorre interrogare la natura

(*) Università degli Studi, Pavia, Italia. E-mail: mceroni@unipv.it 
ed ascoltare sempre e comunque le sue risposte. L'uomo da molto tempo prima di Galileo era stato affascinato dal cielo stellato, dall'alternarsi della notte e del giorno, delle stagioni. I più grandi spiriti dell'umanità, in particolare i Greci che avevano scoperto l'uso sistematico della ragione e la sua applicazione alla conoscenza del mondo, avevano ideato interpretazioni dei moti del sole, della terra, dei pianeti, delle stelle, ingegnosi e basati anche sulla matematica. Aristotele ha finito per determinare la visione dell'universo in tutto il mondo occidentale. Tolomeo aveva semplicemente arricchito e precisato anche dal punto di vita matematico la visione di Aristotele. Eppure i Greci che avevano inventato la geometria e l'aritmetica, che sapevano perfettamente che la terra è rotonda e ne avevano misurato il raggio, non scoprirono la scienza, non arrivarono a varcare la soglia della scienza.

Quando Galileo puntò per la prima volta lo strumento che si era costruito per scandagliare il cielo, il cannocchiale, le cose emersero nella loro evidenza: le lune di Giove erano lì davanti ai suoi occhi, la superficie della luna appariva così come era, non una perfetta sfera come richiesto dalla teoria, ma per come era fatta. Quasi ovunque puntasse il cannocchiale emergevano cose nuove, scoperte affascinanti. Non contava il ragionamento astratto, la bellezza della teoria, occorreva molta più umiltà, conveniva cercare strumenti adatti per cogliere le cose come erano veramente, non come l'immaginazione dell'uomo di quel tempo le pensava. La scienza dunque ha bisogno di evidenza; se non si arriva all'evidenza occorre trattenere il giudizio, ammettere la propria ignoranza, cercare un modo nuovo per raggiungere l'evidenza.

Ma diciamo la parola più importante e chiara che segna la nascita della scienza, è la parola misura. E infatti è attraverso le misure ripetute che in molti casi si raggiunge l'evidenza. Lo scienziato che cerca di capire un fenomeno, cioè etimologicamente l'aspetto che si manifesta ai nostri sensi di una determinata "cosa", compie misure, le ripete, varia le condizioni sperimentali e poi misura nuovamente, cerca l'evidenza che l'ipotesi interpretativa che aveva emesso si mostri corretta, consenta di prevedere gli effetti e addirittura può giungere a scrivere una legge matematica in grado di descrivere il fenomeno e di prevederne le variazioni. In questo consiste il metodo scientifico: la sua applicazione leale permette di procedere anche se lentamente, non senza errori e correzioni, deviazioni dalla strada maestra e ritorni ad essa, di arricchire e sviluppare la nostra conoscenza del mondo, 
delle cose. L'evidenza scientifica non è mai assoluta, perché nuove misure, nuovi strumenti di indagine e di misura possono approfondire, chiarire, persino cambiare la conoscenza accumulata.

Fa parte della scienza la tendenza davanti ai fenomeni della natura di scomporli, di operare semplificazioni, di considerare singoli aspetti (riduzionismo metodologico della scienza) per poter applicare ad essi misure, coglierne le leggi, per poi tornare a ritroso a metterli insieme, a considerarne le implicazioni, le interazioni che stanno alla base dell'intero fenomeno, tesa a ipotizzare una teoria che riesca a spiegare un numero sempre maggiore di aspetti e di fenomeni, a tener conto della totalità degli aspetti che compongono un determinato fenomeno naturale.

Potremmo a questo punto chiederci quali sono i limiti di applicabilità della scienza. Ritengo sia difficile, se non impossibile rispondere a questa domanda in modo chiaro e definitivo. Noi non possiamo prevedere ora quali saranno gli sviluppi futuri della scienza. La scienza quale parte fondamentale della conoscenza, della cultura umana condivide una caratteristica intrinseca e peculiare dell'uomo: per sua natura lo sforzo conoscitivo dell'uomo tende a conoscere tutto, ad andare fino al fondo delle cose, a penetrare ogni mistero, a mutarlo in problema da affrontare e chiarire, da dipanare. E questo è quello che sta accadendo nel campo della fisica: essa cerca di elaborare teorie che possano spiegare in modo unitario, razionale, chiaro la totalità dell'universo. Tuttavia proprio la nostra cultura razionalista, con Kant, ha chiarito che la conoscenza dell'uomo non potrà mai raggiungere e dipanare "le cose in sé", noi non possiamo raggiungere la conoscenza totale delle cose come sono al loro fondo, perché non siamo noi che le abbiamo fatte, anzi ne dipendiamo. Pertanto la scienza che è figlia del razionalismo moderno non può avanzare la pretesa di arrivare a conoscere tutto. Se lo fa contraddice prima di tutto se stessa, perché essa è leale discepola della natura, della realtà ed è proprio quest'ultima ad essere irraggiungibile nella sua totalità, nella sua essenza, nella sua fattura ultima. "Chi non riesce più a provare stupore e meraviglia è già come morto e i suoi occhi sono incapaci di vedere" ha scritto Einstein.

Conviene affrontare il problema dei limiti di applicabilità della scienza da un punto di vista più operativo e chiaro. Comunque la scienza si applica allo studio di quegli aspetti della realtà che sono definibili univocamente e che si possono misurare o osservare ripetutamente. Gli scienziati possono essere tentati di ritenere che solo gli 
aspetti ben definibili e misurabili sono reali e che tutto ciò che non ha queste caratteristiche è irrilevante o addirittura inesistente, frutto solo della fantasia umana. Vorrei subito sottolineare che tale affermazione, pur se fatta da scienziati esemplari in campo scientifico, non ha nulla a che vedere con la scienza, è l'affermazione di una propria visione delle cose, che non esprime una conoscenza scientifica. La scienza non si occupa della natura ultima delle cose, ma cerca pazientemente e umilmente di dipanare la conoscenza di ciò che può afferrare e misurare, cioè in ultima analisi di capire il meglio possibile quale sia il meccanismo con il quale avvengono i fenomeni.

\section{MA CHE COS'È LA COSCIENZA?}

La parola coscienza non è definibile in modo univoco, chiaro, completo. Essa è la parola moderna, usata sistematicamente da Cartesio, che ha penetrato tutta la nostra cultura, per indicare la caratteristica essenziale, fondamentale dell'uomo, del soggetto umano, dell'Io (anche la parola soggetto ha a che fare con questo). In passato si usavano le parole "anima", "spirito" con gli equivalenti in greco "psiche", "pneuma" (cioè respiro).

E' immediatamente chiaro a tutti che è veramente difficile definire la natura ultima dell'uomo, la sua essenza, la sua caratteristica unica rispetto a tutte le cose, ma anche a tutti gli esseri viventi di questa terra. Per poter definire la coscienza devo usare la coscienza! Così ci troviamo in questa curiosa situazione di dover usare dell'essenza di noi stessi per definire noi stessi. Se comunque tentiamo qualche definizione, ad esempio che la coscienza è la capacità di renderci conto della realtà e di noi stessi, oppure che è quella capacità per cui la realtà e noi stessi sono presenti a noi stessi, oppure che è quel livello della natura in cui le cose e noi stessi diveniamo trasparenti dentro la nostra esperienza, ci accorgiamo subito che usiamo parole diverse, ma che dicono la stessa cosa: inesorabilmente usiamo delle tautologie, come dicessimo in fondo la coscienza è la coscienza.

La parola coscienza allude anche a quella esperienza che abbiamo giornalmente di abolizione della nostra coscienza nel sonno e di recupero della stessa al risveglio. Nella veglia le cose e noi stessi siamo presenti a noi stessi, nel sonno è come se le cose e noi stessi non esistessero, ma ovviamente esistono, solo che noi non ne siamo coscienti. 
Pensate che la coscienza fa sì che la totalità di me, cioè tutto il mio passato, tutte le mie esperienze di tutta la mia esistenza sono presenti nell' istante presente in cui solo vivo, insieme con tutte le mie aspirazioni, i miei progetti, le mie aspettative per il futuro. E il miracolo si ripete all'istante successivo in modo nuovo perché si è aggiunta l'esperienza dell'istante precedente.

Si capisce bene come la coscienza sia sfuggita ad ogni tentativo di essere afferrata da metodologie scientifiche e quindi molte correnti neuroscientifiche hanno cercato di negarla, o di eliminarla, o di ridurla a strano epifenomeno, non essenziale per la vita dell'uomo. Cioè l'uomo moderno, l'uomo razionale, occidentale, che ha scoperto la scienza e imposto la propria cultura a tutto il mondo, lo stesso uomo che ha esaltato la grandezza della coscienza, ora è giunto al punto di volerla eliminare, di voler negare ciò che rende l'uomo uomo, di negare se stesso!

Il tema in questione viene affrontato da Savoldi nell'opera monumentale sulla Coscienza in un paragrafo della Introduzione sotto il titolo di "una ripresa pascaliana". Così scrive Savoldi: "L'antagonismo tra coscienza cognitiva e coscienza fenomenica potrebbe essere letto sul registro pascaliano come l'antagonismo tra l'esprit de finesse e l'esprit de géométrie (Pascal B, Discours sur les passion de l'amour, Pansées et opuscules, Hachette, Paris). Nell'esprit de géométrie i principi sono palpabili, ma lontani dall'uso comune, difficili da vedere, ma una volta visti è impossibile che possano sfuggire. Nell'esprit de finesse i principi sono nell'uso comune davanti agli occhi di tutti. Non è necessario volgere la testa né farsi violenza per vederli; ma è necessario avere una buona vista perché i principi sono così sciolti (delié) e in così grande numero che è quasi impossibile che qualcuno non sfugga. Ora, l'omissione di un principio induce all'errore, così bisogna avere la vista molto buona per vedere tutti i principi e lo spirito giusto per non ragionare in modo falso sui principi noti. Tutti gli spiriti geometrici sarebbero fini se avessero la vista buona, perché essi non ragionano in modo falso sui principi che essi conoscono e gli spiriti fini sarebbero geometrici se potessero piegare lo sguardo verso i principi loro estranei della geometria. Ciò che fa sì che certi spiriti fini non siano geometrici è il fatto che essi non possano volgersi verso i principi della geometria; mentre ciò che fa sì che i geometrici non siano spiriti fini è il fatto che essi non vedano ciò che è davanti a loro e che abituati ai principi netti e grossolani della geometria, ragionino solo dopo aver visto e manipolato i loro principi; essi si perdono nelle cose della finezza dove i principi non si 
lasciano manipolare; ci sono cose talmente delicate e numerose che occorre un senso molto delicato e netto per sentirle e giudicarle in modo giusto secondo questo sentimento, senza poterli dimostrare con ordine come la geometria, perché non se ne possiedono i principi. E' necessario vedere la cosa con un solo sguardo e non attraverso i progressi del ragionamento almeno fino a un certo grado; si può esprimere "esattamente la differenza stabilita da Pascal tra l'esprit de finesse e l'esprit de géométrie, scrive con ragione Nicola Abbagnano; il primo comprende mentre il secondo ragiona." (N Abbagnano, Storia della Filosofia, vol $2^{\circ}$ pag 258). In effetti, è necessario un certo grado di finezza per approfondire il ragionamento geometrico; ma è evidente che lo spirito di finezza ha come oggetto il mondo degli uomini, mentre lo spirito geometrico ha per oggetto il mondo esterno".

Quando si ascoltano affermazioni come "la coscienza è integralmente prodotta dal cervello", "se arriveremo a decifrare il funzionamento del cervello potremo capire esaurientemente la coscienza e tutte le sue manifestazioni”, affermazioni di cui sono piene tutte le pagine scientifiche dei giornali e delle riviste e che vengono ritenute dimostrate, chiare e acquisite, si resta veramente meravigliati. Infatti tali affermazioni non sono scientifiche perché mettono insieme fenomeni completamente diversi: il cervello, parte del corpo umano, indagabile con metodi biologici, fisici, chimici e la coscienza, che, come abbiamo visto, non si riesce a definire, a precisare e che pertanto la scienza non può afferrare nella sua totalità. Quelle affermazioni sono completamente prive di un supporto sperimentale e di un rigore logico, che permetta di passare da una causa ad un effetto. Le neuroscienze sono interessate e impegnate a chiarire il fine funzionamento del cervello e di tutte le sue straordinarie capacità e si fermano, consce della applicabilità dei propri metodi, davanti alla coscienza, cioè, come abbiamo visto, a ciò che indica il mistero ultimo dell'uomo anche davanti a tutte le altre realtà viventi del mondo, la sua unicità e assoluta particolarità.

Ogni altra posizione cade nella dimenticanza dell'esprit de finesse, cioè nella cecità fino alla negazione di ciò che è comunque evidente all'uomo semplice della strada, libero dal pregiudizio imposto dalla cultura di massa, a ciò che sta davanti agli occhi di tutti e di cui tutti fanno uso per vivere: la coscienza è una caratteristica squisitamente umana e straordinaria che rende l'uomo unico nell'universo, per quel che fino ad ora sappiamo, a essere capace di consapevolezza delle cose e di sé.

Ecco dunque in cosa consiste la difficoltà delle neuroscienze 
davanti alla coscienza: "ciò che fa sì che gli spiriti geometrici non siano spiriti fini è il fatto che essi non vedano ciò che è davanti a loro".

Il fenomenologo americano Hubert Dreyfus nel suo libro del 1972 "What computers can't do" afferma: "Se il paradigma del computer diventa così forte che gli uomini cominciano a pensare a se stessi come se fossero dei dispositivi digitali, fatti sul modello delle macchine dell'intelligenza artificiale, allora, dal momento che le macchine non possono essere come gli uomini....., gli esseri umani possono progressivamente diventare come macchine. Il rischio non è l'avvento del computer superintelligente, ma di esseri umani sottosviluppati intellettualmente".

E' esattamente quello che dice Pascal: si comprendono le sottigliezze della fisica quantistica, ma non si vede più quello che è davanti a noi.

BIBLIOGRAFIA

Savoldi F, Ceroni M, Vanzago L. "La Coscienza”, Ed. ARAS, Fano Perugia, 2013, pag. 27-28. 